\title{
Out of pocket payment, affordability and availability of essential medicines in Africa: systematic review
}

\begin{abstract}
Background: Availability and affordability of medicines are key determinants of universal health coverage, yet achieving them presents a major challenge especially in low-income countries. This study aims to overview the evidence on the accessibility of essential medicines in the African continent.

Methods: A quantitative literature search published in English since 2014 was held from valid databases; such as, Scholar, Economic Literature, Global Health, PAIS International, and African Index Medicus. The search was erperformed from September 16 to 20, 2019. Two authors (G.A and D.T) screened the titles, reviewed the articles for inclusion, extracted the data, and conducted a quality assessment of the literature. The third author (N.A) commented on the review. We have used the universal definition of OOP, availability, and affordability.

Results: Of 34, 06 articles initially identified, 19 were eligible for inclusion. These were cross-sectional and case-control household and health facility studies published in 2014 in Africa. They demonstrated that the availability of some essential medicine (antibiotics) $>80 \%$ met WHO's target. However, drugs for non-communicable diseases show unavailable that range within $20.1 \%$ to $60.8 \%$. Households access health services mainly through OOP. An item patients' expense more for, was fees for drugs (62.3\%) (Congo) and was an expensive component of expenditure in private and public health facilities with a mean of 16.7USD and 25.5USD, respectively (Burkina-Faso). Drugs for non-communicable diseases were the most expensive than drugs for infectious diseases (median $=0.62$ USD) (Ethiopia). The majority of core essential medicines in private and public outlets are unaffordable. There was a considerable variation in the affordability of basic treatment for infectious and non-communicable diseases. Interestingly, the potential source of inadequate availability of essential medicines and the presence of unaffordability was not investigated in Africa.

Conclusion: Evidence suggests that even though, African countries show progress in coverage and affordability for some core essential medicines, it tills needs an effort to convey the WHO's goal particularly for drugs of non-communicable disease. Future studies need to explore reasons for the persistent unavailability, unaffordability as well as high OOPs for medicines.
\end{abstract}

Keywords: availability, out of pocket payment, affordability, essential medicines, access medicines, Africa
Volume 10 Issue 2 - 2021

\author{
Getahun Asmamaw,' Dinksew Tewuhibo, ${ }^{2}$ \\ Nardos Asffaw ${ }^{3}$ \\ 'Department of Pharmacy, Arba Minch University, Ethiopia \\ ${ }^{2}$ Department of Pharmacy, Medawolabu University, Ethiopia \\ ${ }^{3}$ Pharmacist, Addis Ababa, Ethiopia
}

Correspondence: Getahun Asmamaw, Department of Pharmacy, Arba Minch University, Arba Minch, Ethiopia, Email getahun.asmamaw@amu.edu.et

Received: December 02, 2020 | Published: March 16, 202 |
Abbreviations: GAP, global action plan; HAI, health action international; LIC, low-income countries; MIC, middle-income countries; NCD, non-communicable diseases; NEML, national essential medicines lists; OOPEs, out of pocket payment expenditures; OPP, out of pocket payment; PRISMA, preferred reporting items for systematic review and meta-analysis; SES, socio-economic status; WHO, world health organization

\section{Introduction}

Essential medicines are aimed to satisfy the priority health care needs of the population. Using an essential medicine list (EML) makes medicine management easier in all respects; procurement, storage, and distribution are easier with fewer items, and prescribing and dispensing are easier for professionals. ${ }^{1}$ States have a legal obligation to make essential medicines accessible to those who need them at an affordable cost. Determining the degree of affordability of medicines, especially in low- and middle-income countries (LICs and MICs), is an important, yet complex undertaking as affordability is a vague concept. ${ }^{2}$ Affordability is not straightforward to define merely. The WHO and HAI methodology is widely used to measure affordability. Affordability the number of daily wages needed to obtain a standard treatment by the lowest-paid unskilled government worker. This could clearly show the reality of how the medicine payment to revenue daily wage for who must pay out-of-pocket for medicines. ${ }^{3-5}$ In lower-income countries, medicine cost takes a great portion of total healthcare cost. Unfortunately, medicines provided free in the public health sector are often unavailable, ${ }^{6}$ Out of pocket medicine payment is the main means of accessing healthcare service in African countries, comprise the main method in health care financing. ${ }^{7}$ Consequently, where medicine prices are high, people may be unable to procure them and therefore forego treatment or they may go into debt. For this reason, WHO has designated affordable prices as a determinant of access to medicines (together with rational selection and use, sustainable financing, and reliable health and supply systems). ${ }^{5}$

In the national drug policy of countries, ensuring the availability and affordability of essential medicines are often placed as the main objective. Suggesting a challenge for the national drug policy promise. To achieve this, many low/ middle-income countries have National 
Essential Medicines Lists (NEMLs). ${ }^{8}$ In African countries, especially in rural areas, large stock-outs of essential drugs are common in most health facilities. In terms of equity, evidence has confirmed that the presence of disparities by type of institution, managerial body, and urban-rural residence. ${ }^{9}$ The goal of universal health coverage is challenging for chronically under-resourced health systems. Although household out-of-pocket payments are the most important source of health financing in low-income countries..$^{10}$ Due to the importance of the need to access medications and lack of pooled data of determinants for inaccessibility of medication in Africa; this systematic review aimed to assess the extent of availability, out of pocket payment, and affordability and their burden on the accessibility of medications in the African continent.

\section{Methods}

\section{Eligibility criteria}

Quantitative studies, studying the availability, out of pocket payment, and affordability in any of African countries published from 2014 to 2019 in the English language were imposed. This was because findings can be generalized from quantitative studies than qualitative studies. The need to take only 5-year data is that recent data can able to represent more of the current practice. Studies whose primary or secondary outcomes are either of OPP/availability/ affordability were included. Since more relevant and clear data can be oufound from those studies. Moreover, retrospective and prospective studies were included to widen the domain of studies to be included. Using PICO's framework (Population, Intervention, Comparison, Outcome) for the literature search, our sampling population was defined as any individuals or households that use private or public health facilities in Africa. The intervention being examined was OPP, affordability, or availability status of essential medicine. Our primary outcomes were defined as OOPs, which being defined from the patient's perspective: i.e. payments made by individuals or households, to health facilities for medication. Another primary outcome was availability which is defined as the availability of core medicines at the facility per number of key medicines available in a facility. Affordability was defined as the number of days' wages needed by the lowest-paid unskilled government worker to purchase standard treatments.

Studies were excluded based on their titles and abstract if considered not relevant to the topic. It is considered as not relevant if the title/abstract does not contain keywords, beyond the current review objective and if it is a unrelated title. Studies were also excluded after a full-text review which was estimated to be low quality. Studies not in Africa and not published in English were also excluded from this review. Because We were interested in quantitative studies only, we had excluded technical notes, case reports, and literature reviews. Focus groups, stakeholder analyses. Studies with no clear aims or objectives, no statistical analysis, or that were not peer-reviewed were also excluded. Studies also excluded for this review, those assed the out of payment, affordability, or availability status of essential medicine from the government payer perspective.

\section{Information sources and search strategy}

We did a systematic review using the Preferred Reporting Items for Systematic Reviews and Meta-Analysis (PRISMA) guidelines. ${ }^{11}$ Two authors G.A and D.T have searched PubMed, Google Scholar, Economic Literature, Global Health, PAIS International, and African Index Medicus for articles published since 2014. We have used the following search terms to search all trial registers and databases: "Out of Pocket Payment OR Availability OR affordability OR catastrophic health OR national health" AND "Essential medicines". This was combined with terms for the country: "Africa or African" using the conjunction "AND". The same search was conducted in all seven databases.

\section{Screening of studies}

Records identified from various electronic databases, indexing services, and directories were exported to END-NOTE reference software version 8.2 (Thomson Reuters, Stamford, CT, USA) with compatible formats. Duplicate records were identified, recorded, and removed with ENDNOTE. Some duplicates were addressed manually due to variation in reference styles across sources. Thereafter, WE have screened the title and abstracts with predefined inclusion criteria. The identification of records, screening of titles and abstracts as well as evaluation of eligibility of full texts for final inclusion was conducted per the Preferred Reporting Items for Systematic Review and Meta-analysis (PRISMA) flow diagram. ${ }^{11}$ PRISMA checklist ${ }^{12}$ was also strictly followed while conducting this systematic review.

\section{Data collection process}

Data collection was completed by reviewers called (G.A) and (D.T) using a standard data extraction form (Table 1). We have collected general information about the articles: the study citation, authorship, year, and type of publication. Study characteristics included study objectives, design, data source, sampling technique, power calculation, and study setting. We collected information regarding the participant characteristics, including a description of the study population, the inclusion of socioeconomic status (SES) in the analysis, and population size. Outcomes were availability, affordability, and OOPEs. For the statistical analysis, we have collected the odds ratios, P-values, confidence intervals, and the definitions of the variables used in the authors' analysis that compared differences between the primary outcome of this review. To assess the quality of the articles meeting the inclusion criteria, we have used a checklist similar to that used by Mirza and Jenkins. ${ }^{13}$ The checklist (Table 4) included eight quality items: 1) Explicit study aims stated; 2) Sample size justification is given; 3) Representative sample or justification; 4) Clear inclusion and exclusion criteria; 5) Reliability and validity of measures justified; 6) Adequate description of the data; 7) Statistical significance assessed; 8) Discussion on the generalizability of the study provided. One point was given for a "yes" answer and none for a "no" answer, for a possible maximum score of eight points. The total score is the average score for the quality items based on each author's review. Scores less than 5 were considered low, 5-6 moderate, 7-8 high in quality. Studies scored less than 5 were excluded and studies considered as moderate and high quality were included for this review.

\section{Results}

A total of 3,406 articles were initially identified; 478 articles with duplicates were excluded, and Articles were excluded $(\mathrm{N}=2,885)$ Because of their title (Unrelated title) $(\mathrm{N}=2,799)$, abstract $(\mathrm{N}=98)$, inclusion and exclusion criteria $(\mathrm{N}=12)$ (Figure 1). Nineteen articles were eligible for full-text review of which met our inclusion criteria. Table 4 summarizes the results of the quality assessment of the studies conducted by a single independent reviewer. Table $2 \& 3$ provide a general description of the studies included: study aims/objectives, study population and size, study design, setting, statistical analysis, and key findings. Studies that met our inclusion were from Uganda (4), Ethiopia (3), Mozambique (1), Nigeria (2), Kenya (4), Malawi (1), Burkina Faso (1), Congo (1), Cameroon (1), and Ghana (2). They were cross-sectional studies (surveys) conducted across 18 
out of 19 studies in Africa. The remaining single study was a casecontrol study from Nigeria. 18 studies collected primary data in the form of a questionnaire administered to individuals or households. The $19^{\text {th } 14}$ analyzed secondary data, i.e., 10 districts across the country 209 Ugandan health facilities to demonstrate that SARA data can be used to model the availability of essential medicines for treating no communicable diseases (EM-NCD). All nineteen studies asked participants to recall information associated with seeking healthcare from five days to twelve months preceding the surveys. Respondents were caregivers of all members of the households. The study populations ranged from 135 individuals to approximately 3341 households. Private and public health facilities were ranged from 6 to 600. Furthermore, essential medicines assessed in these studies were range from 6 to 37 items in range to 397 drug formulations. Most of the studies examined drugs for infectious diseases (3), noncommunicable diseases (7), mental healthcare (1), child; reproductive and maternal health (2), antineoplastic medicines (1), and the rest studies revealed their finding on any medicine available or buy during the study.

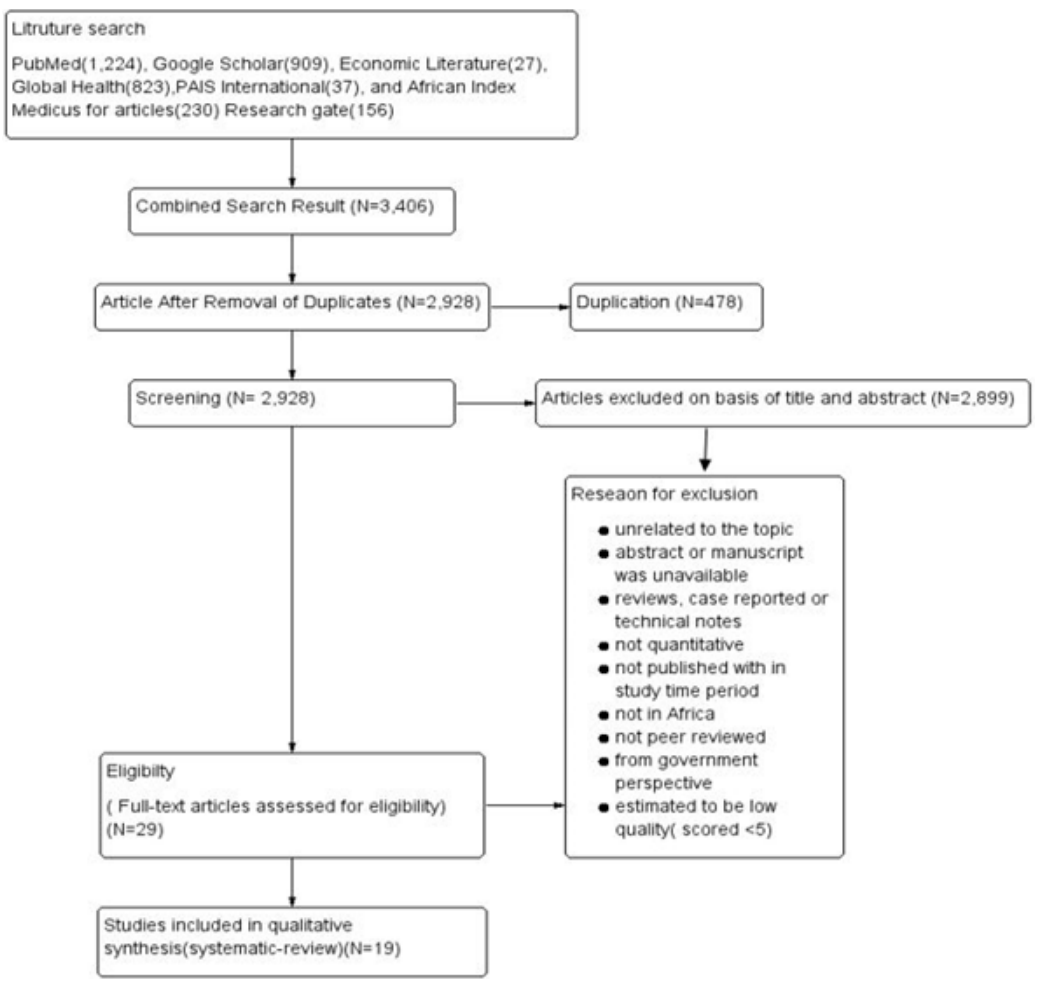

Figure I PRISMA flowchart of study selection.

\section{Availability}

Around 12 studies out of 19 revealed the availability of core essential medicine as their primary or secondary outcome. Both public and private health facilities have a comparable contribution to the availability of Essential medicines. Except for ${ }^{15}$ study, all of them listed drugs specifically even though the item differs according to their objective of the research question. Generally, concerning availability across countries Ghana and Kenya having better availability than Uganda. ${ }^{16}$ Public health facilities have more drugs for infectious, most NCD, and mental health than private outlets. But private health facilities had more drugs for maternal/reproductive health and anticholinergics comparing to public ones.

\section{Antibiotics}

Two studies evaluated the availability of antibiotics ${ }^{17,18}$ which ranged from $92.3 \%$ and $98.5 \%$, in private and public pharmacies respectively in one study in Ethiopia. ${ }^{18}$

\section{Non-communicable diseases}

Among 7 studies evaluated essential drugs for non-communicable diseases, four of them are about availability and three of them were from Uganda. Most available drugs for treating Parkinson's disease were Ergot-derived dopamine agonists (DAs) and anticholinergics were available in 37 (89.5\% private) outlets and 35 ( $84.4 \%$ private) outlets, respectively ${ }^{19}$ in Kenya. A study done in Uganda revealed the availability of the medicines ranged from $20.1 \%$ for unfractionated heparin (UFH) to $100 \%$ for oral hypoglycemic agents (OHA. ${ }^{20}$ The other study in the same country evaluated the availability of inhaled short-acting beta-agonists (SABA), oral leukotriene receptor antagonists (LTRA), inhaled LABA-ICS combinations and inhaled corticosteroids (ICS) in all the study sites was $75 \%, 60.8 \%, 46.9 \%$, and $45.4 \%$ respectively ${ }^{20}$ that For-profit facilities' EM-NCD counts were $98 \%$ higher than public facilities $(\mathrm{p}<.001)$.

\section{Mental healthcare, reproductive and maternal health}

Thioridazine was the most commonly available antipsychotic across all facilities (9 of 24, 37.5\%) in Mozambique. ${ }^{21}$ In Uganda, the most available commodity was oxytocin injection ( $86 \%$ in mission facilities and $84 \%$ in public facilities. ${ }^{22}$

\section{Antineoplastic}

One study done across African 32 countries reported that antineoplastic ranged from $57.7 \%$ and $8.3 \%$ at public and private outlets respectively for antineoplastic in multi-country studies. ${ }^{15}$ 


\section{Out of pocket payment}

Six out of nineteen studies reported pocket payment. OOP was the principal payment mechanism of households. The most important expense item was fees for drugs and medicines $(62.3 \%)$ (Congo). ${ }^{9}$ The medication was the most expensive component of expenditure in both public and private facilities with a mean of 16.7USD and (25.5USD), respectively ${ }^{15,23}$ in Burkina Faso and other 32 African countries. The medicines cost was calculated to be (median $=0.62 \mathrm{USD}, \mathrm{IQR}$ $=0.00-1.74)$ (Ethiopia).${ }^{24}$ Drugs for non-communicable diseases were the most expensive than drugs for infectious drugs. Enalapril and hydrochlorothiazide were the most expensive combination of all prescribed antihypertensive medicines attracting a mean annual cost of US\$ 43.7 (95\% CI, 29.2-58.3) (Kenya). ${ }^{25}$ Over three-fifth (69\%) of the respondents paid out-of-pocket for health services and about fourfifth $(76.9 \%)$ of the respondents did not budget monthly for health care services (Nigeria). ${ }^{26}$

\section{Affordability}

Seven studies revealed their result as affordability as a primary and secondary outcomes. All of them calculate affordability as obtained unit retail prices of the medicines in USD were converted to a median price ratio (MPR) by dividing the median local price by an international reference price (IRP). Three of seven studies reported medicines they had assessed are affordable ${ }^{17,19,27}$ and the other two studies $^{18,28}$ reported medicines are unaffordable. The remaining two studie $^{20,28}$ also reported both but among different drug items. A study done in Malawi of essential medicines is affordable which the median price ratio (MPR) to a wholesale international procurement price was 2.8 in public health facilities and even lower in the private sector (MPR 2.3) ${ }^{27}$ Only five outlets considered L-dopa affordable, all of which sold 100 tablets for less than $\$ 31 .{ }^{19}$ MPR for lowest price medicines were found to be 1.18 times their IRP in the public sector. ${ }^{17}$ Affordability for inhaled salbutamol was 2.2 days' wages. ${ }^{20}$ In comparison, only $7(32 \%)$ of the studied medicines (oral aspirin $500 \mathrm{mg}$, hydrochlorothiazide $50 \mathrm{mg}$, furosemide $40 \mathrm{mg}$, nifedipine 10 and $20 \mathrm{mg}$, Glibenclamide $5 \mathrm{mg}$, and metformin $500 \mathrm{mg}$ ) were affordable according to the study definition of affordability i.e. monthly cost of medicine of $\leq 1$ days' wages of a lowest-paid public servant in the study performed in Western Cameroon. ${ }^{28}$ Metformin and benzathine benzylpenicillin had a median price ratio of 1.5 with statins being largely unaffordable (at least 30.51 days' wages). ${ }^{28}$ MPRs for antibiotics included in the analysis were 4.1 and 2.7, standard treatments for 9 of them $(81.8 \%)$ were unaffordable both in the private and public sectors. ${ }^{18}$ In Uganda, the most unaffordable medicines were Mixtard, simvastatin $20 \mathrm{mg}$, and heparin $5000 \mathrm{IU}$ costing 18.7 days' wages, 30.5 days' wages, and 182.36 days' wages respectively. ${ }^{20}$

\section{Discussion}

Essential medicines are aimed to improve healthcare accessibility through their availability at a right time, in an adequate amount, at the right dosage form, at the right quality, and with the price that client can afford. Our review demonstrates that study areas investigating the availability, out of pocket payment, and affordability are inadequate in most the African countries. However, available research articles show progress was observed in availability, affordability, and out of pocket payment in Africa. Thus, there are challenges in clinical practice. The present review demonstrates that the WHO target $(>80 \%)$ for the availability of essential medicine was not met yet; including generics, both in the public and private health sectors. ${ }^{29}$ The availability of medications for most infectious diseases has been met the goal of WHO in Africa. However, the essential NCD medicines were unavailable at any of the health facilities in the continent.
Moreover, the absence of NCD drugs was more seen in public health facilities. Our findings are in agreement with other reports. For instance, the report from lower-income countries study revealed, only $15.2 \%$ of lowest-priced NCD generic medicines met WHO's target in the public health facilities of Africa. Thus, $18.9 \%$ are available at private health facilities. ${ }^{30}$ The possible explanations for the absence of NCD at public health facilities might be, the commonly African government's problem with procurement, supply chain management, and underfunding of medicine for NCDs. The other possible source might be, due to increasing the noncommunicable prevalence in Africa, the high demand for lower-priced NCD medications at public health facilities. ${ }^{31}$ This could result in patients to look for an alternative for brand products from private ones. Furthermore, peoples might decide not to use healthcare services. Consequently, peoples could face financial burdens due to healthcare seeking.

Generally, the availability of essential medicines shows progress from 2014 to 2019. The reforms of the health system among African counties, the attention is given by WHO, and other concerned bodies in the implementation of universal health coverage (UHC) might be played a major role. Interestingly, both public and private health facilities were contributed to the availability of medicines that are consistent with WHO's recommendation. Implying the likelihood of facing difficulties to access healthcare in Africa is getting improved. The present study illustrated that medicines are the most expensive among healthcare services. For instance, a cross country survey in Africa reported, medication was the most expensive component of expenditure in both public and private facilities with a mean of 16.7USD and (25.5 USD), respectively. ${ }^{15,23}$ Other studies were done in Tehran also yconsistent with our finding that drug for chronic disease is expensive..$^{10}$ This implies, Africa ought to set or implement financial protection policy i.e. health insurance, borrowed money to solve health service access barriers. Hence, Consistent pieces of evidence have also shown that reduction or removal of out-of-pocket payment at the point of use enhances the utilization of health care services. ${ }^{32}$

This review revealed that OOP was the principal payment mechanism of households. It complies with a report that $60 \%$ of countries at incomes below $\$ 1,000$ per capita, out-of-pocket spending is $40 \% .^{33}$ This is a very common cause in the middle to lower-income countries. ${ }^{34}$ In Some African countries (Nigeria) factors have been tried to trace several factors especially the gross underfunding of the health sector. Solutions to resolve high pocket payment, studies suggest that disaggregated mixture of health financing, including government budgetary allocation, health insurance (social and private), external funding, and private out-of-pocket spending to finance health care. ${ }^{7}$ Pre-payment via health insurance is seen as one of the viable options that are available to broaden sources of health care financing and hence reduce the dependence and pressure on the government budget. ${ }^{35}$

This review used defined affordability as; drug said to be affordable if the monthly cost of medicine of $\leq 1$ days' wages of the lowest-paid public servant in the study. According to our review, the majority of core essential drugs available in private and public outlets are unaffordable. A study was done in Sudan ${ }^{36}$ and Another systematic review on asthma and COPD in African countries medicines revealed similarities. ${ }^{37}$ Variation of affordability of basic essential drugs for chronic and most prevalent diseases seen in Africa. This variation can cause the challenge to combat the prevalent diseases in the continent. For instance, reasonable affordability is needed to curb this healthcare challenge. The results illustrate that substantial proportions of the population would be pushed into poverty as a result of medicine procurement, implying that in many countries affordability of these treatments is low. 


\section{Limitations}

Some significant limitations of this systematic review are brands that were not taken into account. Secondly, availability only estimated data of per-see, not trend or experience. The affordability metric does not include other healthcare costs. Due to the search strategy, findings that is not from direct titles may be missed. Moreover, some important studies might be missed since the reviewer is single.

\section{Conclusion}

This study shows that despite some impact in enhancing the availability of some essential medicines especially antibiotics; the availability of drugs for non-communicable disease, out of pocket and affordability, remains catastrophic for many people in Africa. Further investigations are needed to explore reasons why affordability and OOPEs persist, particularly for medicines. Furthermore, Evaluating the impact of the health insurance scheme in African countries may key to improve health-care accessibility in Africa. Moreover, affordability and OOP burden should be studied through the category of age, residence, and age group mainly for drugs of chronic diseases.

\section{Acknowledgments}

None.

\section{Conflicts of interest}

The author declares there is no conflict of interest.

\section{Funding}

None.

\section{Authors' contributions}

GA designed the study. GA and DW collected scientific studies, assessed the quality of the study, extracted and analyzed the data. NA commented on the review. GA also prepared the manuscript for publication. All authors have read and approved the manuscript.

\section{References}

1. WHO Policy. Promoting rational use of medicines: core components Patient Care Indicators. Geneva, 2016.

2. LR Cameron A, Ewen M, Ross Degnan D. Medicine prices, availability, and affordability in 36 developing and middle income countries," a Second. Anal Lancet. 2009;373:240-249.

3. van Mourik MS, Cameron A, Ewen M. Availability, price and affordability of cardiovascular medicines: a comparison across 36 countries using WHO/HAI data. BMC Cardiovasc DisordOn. 2010;10(25).

4. Gelders S, Ewen M, Noguchi N, et al. Availability and affordability. An international comparison of chronic disease medicines. WHO EMRO/HAI. 2006.

5. WHO. World Health Organization Access to medicines. 2008.

6. A Cameron, E Van De Poel, M Ewen, et al. Quantifying the Impoverishing Effects of Purchasing Medicines: A Cross-Country Comparison of the Affordability of Medicines in the Developing World. IDEAS. 2010;7(8).

7. NM Marzban S, Rajaee R, Gholami S, et al. Study of Out-of-Pocket Expenditures for Outpatient Imaging Services in Imam-Khomeini Hospital in 2014. Electron Physician. 2015;7(4):1183-1189.

8. UG Assembly. Political Declaration of the High-level Meeting of the General Assembly on the Prevention and Control of Non-communicable diseases. United Nat. New York, 2012.
9. S Laokri, R Soelaeman, DR Hotchkiss. Assessing out-of-pocket expenditures for primary health care : how responsive is the Democratic Republic of Congo health system to providing financial risk protection? BMC Health Serv Res. 2018;1-19.

10. M Zarif-yeganeh, M Kargar, A Rashidian. Out of Pocket Payment and Affordability of Medication for Geriatric Patients in Tehran, Iran. Iran J Public Health. 2019;48(6):1124-1132.

11. A Liberati. The PRISMA Statement for Reporting Systematic Reviews and Meta-Analyses of Studies That Evaluate Health Care Interventions : Explanation and Elaboration. PLos Med. 2009;6(7):e1000100.

12. GP Moher D, Liberati A, Tetzlaff J, et al. Prisma 2009 checklist. 2009.

13. JR Mirza I. Risk factors, prevalence, and treatment of anxiety and depressive disorders in Pakistan: systematic review. BMJ. 2004;328(7443):794.

14. Chankova S, Sulzbach S, Diop F. Impact of mutual health organizations: evidence from West Africa. Health Policy Plan. 2018;328(7443):264-276.

15. M Armstrong-hough, SP Kishore, S Byakika, et al. Disparities in availability of essential medicines to treat non-communicable diseases in Uganda : A Poisson analysis using the Service Availability and Readiness Assessment. POLS ONE. 2018;1-12.

16. R Cherny. SPECIAL ARTICLE ESMO International Consortium Study on the availability, out-of-pocket costs and accessibility of antineoplastic medicines in countries outside of Europe. 2018;2633-2647.

17. A Haakenstad, G Ikilezi, J Achan, et al. Pharmaceutical Availability across Levels of Care: Evidence from Facility Surveys Ghana, Kenya, and Uganda. POLS ONE. 2014;1-12.

18. E Sado, A Sufa. Availability and affordability of essential medicines for children in the Western part of Ethiopia: implication for access. $B M C$ Pediatr. 2016;1-8.

19. G Gutema, E Engidawork. Affordability of commonly prescribed antibiotics in a large tertiary teaching hospital in Ethiopia : a challenge for the national drug policy objective. BMC Res Notes. 2018;4-9.

20. J Mokaya, CL Dotchin, WK Gray, et al. The Accessibility of Parkinson's disease Medication in Kenya: Results of a National Survey. Mov Disord Clin Pract. 2016;3(4)1-6.

21. D Kibirige. Access to affordable medicines and diagnostic tests for asthma and COPD in sub Saharan Africa: the Ugandan perspective. BMC. 2017;1-10.

22. BH Wagenaar. The availability of essential medicines for mental healthcare in Sofala, Mozambique. Glob Health Action. 2017;9716.

23. D Kibira, FE Kitutu, GB Merrett, et al. Availability, prices and affordability of UN Commission's lifesaving medicines for reproductive and maternal health in Uganda. Journal of Pharmaceutical Policy and Practice. 2017;4-11.

24. I Beogo, N Huang, MP Gagnon, et al. Out - of - pocket expenditure and its determinants in the context of private healthcare sector expansion in sub - Saharan Africa urban cities : evidence from household survey in Ouagadougou, Burkina Faso. BMC Res Notes. 2016;1-10.

25. FS Teni, Begashaw Melaku Gebresillassie, Eshetie Melese Birru, et al. Costs incurred by outpatients at a university hospital in northwestern Ethiopia : a cross-sectional study. BMC Health Serv Res. 2018;18(1):1-10.

26. M Njoroge, Martin Njoroge, Peter Nguhiu, et al. Patient costs of hypertension care in public health care facilities in Kenya. Int $J$ Health Plann Manage. 2019;34(2):1-13.

27. P Oyibo. Out-of-pocket payment for health services: constraints and implications for government employees in Abakaliki, Ebonyi state, South east Nigeria. Arf Health. 2014;11(3):481-485.

28. F Khuluza, L Heide. Availability and affordability of antimalarial and antibiotic medicines in Malawi. POLS ONE. 2017;1-15. 
29. AM Jingi. Access to Diagnostic Tests and Essential Medicines for Cardiovascular Diseases and Diabetes Care: Cost, Availability and Affordability in the West Region of Cameroon. POLS ONE. 2014;9(11).

30. WHO. Health Action International. Measuring medicine prices, availability, affordability and price components. Geneva, 2008.

31. M Ewen, M Zweekhorst, B Regeer, et al. Baseline assessment of WHO's target for both availability and affordability of essential medicines to treat non-communicable diseases. PLOS ONE.2017;1-13.

32. J Robertson, C Macé, G Forte, et al. Medicines availability for noncommunicable diseases: the case for standardized monitoring. Globalization and Health. 2015;1-6.

33. Emmett B. Abolishing cost recovery in basic health care: A critical reform for Africa. 2019.
34. World Health Report. Health Systems: Improving Performance. World Health Organization. Geneva, 2015.

35. WHO. Strengthening Health Systems to Improve Health Outcomes: Framework for Action. World Health Organization, Geneva; Switzerland. 2007.

36. PS Park E-J, Kwon J-W, Lee E-K, et al. Out-of-pocket Medication Expenditure Burden of Elderly Koreans with Chronic Conditions. Int $J$ Gerontol. 2015;9(3):166-167.

37. A Majid, CÆ Amjad, M Idries. Availability, affordability, and prescribing pattern of medicines in Sudan. Pharm World Sci. 2009;32(2):209-215.

38. D Kibirige, RE Sanya, R Nantanda, et al. Availability and affordability of medicines and diagnostic tests recommended for management of asthma and chronic obstructive pulmonary disease in sub - Saharan Africa : a systematic review. Allergy, Asthma Clin Immunol. 2019;1-11. 